\title{
Protocol Representation Sub-Domain
}

National Cancer Institute

\section{Source}

National Cancer Institute. Protocol Representation Sub-Domain. NCI Thesaurus. Code C93384.

The Protocol Representation sub-domain is intended for those involved in the planning and design of a research protocol. The majority of business requirements have come from those involved in clinical trial protocols. It focuses on the characteristics of a study and the definition and association of activities within the protocols, including "arms" and "epochs". It also includes the definitions of the roles that participate in those activities. 